Citation: Hirt, C., G. Papp, A. Pal, J. Benedek, and E. Szucs (2014), Expected accuracy of tilt measurements on a novel hexapod-based Digital Zenith Camera System - a Monte Carlo simulation study", Measurement Science Technology, 25, 085004 doi:10.1088/0957-0233/25/8/085004.

\title{
Expected accuracy of tilt measurements on a novel hexapod-based Digital Zenith Camera System - a Monte-Carlo simulation study
}

\section{Christian Hirt}

Western Australian Centre for Geodesy \& The Institute for Geoscience Research, Curtin University, GPO Box U1987, Perth, WA 6845, Australia

Email: c.hirt@curtin.edu.au

Currently at:

Institute for Astronomical and Physical and Geodesy \& Institute for Advanced Study Technische Universität München, Germany

\section{Gábor Papp}

Geodetic and Geophysical Institute

Research Centre for Astronomy and Earth Sciences, Hungarian Academy of Sciences, Sopron H-9401, POB 5, Hungary

\section{András Pál}

Konkoly Observatory of the MTA Research Centre for Astronomy and Earth Sciences, Konkoly Thege Miklós út 15-17, Budapest H-1117, Hungary

\section{Judit Benedek}

Geodetic and Geophysical Institute

Research Centre for Astronomy and Earth Sciences, Hungarian Academy of Sciences, Sopron H-9401, POB 5, Hungary

\section{Eszter Szucs}

Geodetic and Geophysical Institute

Research Centre for Astronomy and Earth Sciences, Hungarian Academy of Sciences, Sopron H-9401, POB 5, Hungary 


\section{Abstract}

Digital Zenith Camera Systems (DZCS) are dedicated astronomical-geodetic measurement systems for the observation of the direction of the plumb line. A DZCS key component is a pair of tilt meters for the determination of the instrumental tilt with respect to the plumb line. Highest accuracy (i.e., 0.1 arc-seconds or better) is achieved in practice through observation with precision tilt meters in opposite faces ( $180^{\circ}$ instrumental rotation), and application of rigorous tilt reduction models. A novel concept proposes the development of a hexapod (Stewart platform)-based DZCS. However, hexapod-based total rotations are limited to about $30^{\circ}$ to $60^{\circ}$ in azimuth (equivalent to $\pm 15^{\circ}$ to $\pm 30^{\circ}$ yaw rotation), which raises the question of the impact of the rotation angle between the two faces on the accuracy of the tilt measurement. The goal of the present study is the investigation of the expected accuracy of tilt measurements to be carried out on future hexapod-based DZCS, with special focus placed on the role of the limited rotation angle. A Monte-Carlo simulation study is carried out in order to derive accuracy estimates for the tilt determination as a function of several input parameters, and the results are validated against analytical error propagation. As main result of the study, limitation of the instrumental rotation to $60^{\circ}\left(30^{\circ}\right)$ deteriorates the tilt accuracy by a factor of about 2 (4) compared to a $180^{\circ}$ rotation between the faces. Nonetheless, a tilt accuracy at the 0.1 arc-second level is expected when the rotation is at least $45^{\circ}$, and 0.05 arcsecond (about 0.25 microradian) accurate tilt meters are deployed. As such, a hexapod-based DZCS can be expected to allow sufficiently accurate determination of the instrumental tilt. This provides supporting evidence for the feasibility of such a novel instrumentation. The outcomes of our study are not only relevant to the field of DZCS, but also to all other types of instruments where the instrumental tilt must be corrected. Examples include electronic theodolites or total stations, gravity meters, and other hexapod-based telescopes.

Key words Hexapod, tilt measurement, tilt meter, Digital Zenith Camera System (DZCS), Monte-Carlo simulation

\section{Introduction}

Zenith cameras are dedicated astronomical-geodetic telescopes for imaging of stars around the zenith point in the sky. Such instruments are operated at field stations during night time primarily to determine the direction of the vertical (plumb line). With a history of more than 120 years, zenith cameras were used in the past, e.g., for geographic positioning (e.g., Runge 
1894), for observation of fluctuations in Earth's orientation and rotation (e.g., McCarthy 1976) and gravity field studies (e.g., Torge 2001). Nowadays, zenith cameras - equipped with digital imaging sensors (charge-coupled device, CCD) and other electronic sensors - are deployed as digital zenith camera system (DZCS) for highly-accurate determination of the gravity field (e.g., Hirt and Flury 2008, Hirt and Seeber 2008), or measurement of refraction anomalies (Hirt 2006). For an overview of DZCS instrumental developments see, e.g., Bürki et al. (2004), Hirt (2004), Kudrys (2007), Ogrizovic (2009), Hirt et al. (2010), Halicioglu et al. (2012), Abele (2012), Hanada et al. (2012) and Wang et al. (2014).

A modern DZCS generally features (1) a CCD-telescope combination for star imaging, (2) a Global Navigation Satellite System (GNSS) receiver for time tagging of the star images and (3) a pair of accurate tilt meters (Hirt et al. 2010). The pair of tilt meters is being operated in perpendicular orientation in order to (i) align the optical axis of the DZCS approximately with the direction of the vertical (instrumental levelling), and to (ii) record any residual tilt of the DZCS during the star imaging. This allows a subsequent mathematical correction of the DZCS tilt. To eliminate instrumental zero offsets, DZCS measurements are carried out in two opposite faces which usually differ by $180^{\circ}$ in azimuth. For the rotation of the DZCS between the two faces, some form of rotational unit (bearing, turntable, with or without motorization) is traditionally used, and a tripod with three actuators or adjustable screws is deployed in most instruments for levelling of the telescope (e.g., Bürki et al. 2004, Hirt et al. 2010).

A novel concept for DZCS instrumental levelling and rotation between the two-face measurements was recently developed and presented at the Geodetic and Geophysical Institute (GGI, Sopron) in collaboration with the Institute for Astronomy (Budapest), both members of the Research Centre of Astronomy and Earth Sciences of the Hungarian Academy of Sciences. In this concept, both the rotational unit and the tripod of traditional zenith camera systems are replaced by a hexapod. Hexapods or Stewart-type platforms (Stewart 1965) are platforms with three pairs of actuators (e.g., electromechanical or hydraulic) offering six degrees of freedom in movement. They are routinely used in several industrial applications, ranging from flight simulators to medical surgery. While the use of hexapod platforms is not new in observational astronomy (e.g., Chini 2000, Koch et al. 2009, Pál et al. 2013, Csépány et al. 2014, Vida et al. 2014), its use in geodetic-astronomical applications and incorporation into DZCS has not yet been proposed nor investigated. 
In the concept of a hexapod-based DZCS, the CCD sensor/telescope and tilt meters will be mounted as 'payload' on top of the hexapod platform which - in our case - will be based on six identical electromechanical actuators. Allowing versatile motion and micrometer-precise positioning by length variation of the actuators, a hexapod-based DZCS may offer some interesting potential advantages over "conventional” DZCS. For instance, as a benefit of a hexapod-based DZCS, there will be one unified system deployed for all rotation and tilting of the system rather than a combination of two systems (motorized tripod and rotational unit). Given the high dynamic of hexapod platform positioning, a gain in speed for a DZCS twoface measurement could be possible. As a further potential benefit, star tracking will become possible over short time intervals, enabling more accurate imaging in general and increasing the star count (capturing of fainter stars) in particular. By way of background, DZCS are normally operated as non-tracking instruments where stars are imaged as trails due to Earth's rotation. However, while a possible operation of a hexapod-based DZCS in tracking mode may improve the astrometric imaging, accurate tilt measurements may become more demanding, e.g,. due to accelerations that superimpose the actual tilt signal and have to be removed through filtering.

Different to a rotational unit that allows arbitrary rotation of the DZCS sensors, a hexapod is not capable of executing $180^{\circ}$-rotations. Instead, the maximum possible azimuthal rotation angle between the two faces is - depending on the hexapod design - limited to a range between about $30^{\circ}$ to $60^{\circ}$ which is equivalent to $\pm 15^{\circ}$ to $\pm 30^{\circ}$ in yaw rotation. Thus, the geometry of the two-face measurement with hexapods will be substantially different to a conventional DZCS measurement in opposite faces (i.e., $180^{\circ}$ ). This immediately raises the question of the impact of the rotation angle between the two faces on the accuracy of the tilt measurement.

The goal of the present study is the investigation of the expected accuracy of tilt measurements to be carried out on future hexapod-based DZCS, with special focus placed on the role of the limited rotation angle. The study is organised as follows. Section 2 briefly reviews our mathematical model for the accurate computation of the DZCS tilt correction from tilt measurements in two faces. Section 3 then describes and applies a Monte-Carlo-type simulation in order to derive accuracy estimates for the tilt correction as a function of several parameters, among them the rotation angle between the two faces. This will a.) quantify the expected loss in tilt measurement accuracy when compared to the assumed-to-be-ideal $180^{\circ}$ 
rotation, and b.) clarify if tilt measurements on hexapod-based DZCS can be expected to be sufficiently accurate at all (say few tenths of arc-seconds or better) for state-of-the-art DZCS field operation. Section 4 discusses the findings in view of the future construction of hexapodbased DZCS and draws some conclusions.

The relevance of the findings of this study is not limited to the discipline of geodetic astronomy and astronomic-geodetic instrumentation such as DZCS. The relevance may be given also in view of all other types of instruments where the instrumental tilt must be corrected. Examples include but are not limited to instruments for angle measurements such as electronic theodolites or total stations, but also gravity meters (see, e.g., Torge 2001 for a description of these instruments). However, the models and investigations presented in this study may also prove useful in observational astronomy, where hexapod-based telescopes are being increasingly used (e.g., Koch et al. 2009; Pál et al. 2013), and highly-accurate (say few tenths of arc-seconds or better) tilt measurements appear not yet to be routinely applied.

\section{Tilt measurements and mathematical models}

For the tilt measurement on a DZCS, a pair of tilt meters (two one-axis systems or one twoaxes system) is mounted in (approximately) perpendicular orientation next to the CCDtelescope. In the ideal case the two tilt sensors and the optical axis of the CCD camera defines a rectangular frame which is oriented to the plumb line by means of the tilt sensors. The tilt (misalignement) of the CCD-telescope with respect to the plumb line is measured in two spatial directions which we denote here with $n_{1}$ and $n_{2}$ (Fig. 1a). In practice, tilt readings are always subject to some unknown offset $\Delta n$ (also known as normal point in surveying terminology) which can change as a function of time, depending on factors such as changes in ambient temperature, humidity or pressure (e.g., Hirt and Kahlmann 2004). To determine and remove the unknown offsets ( $\Delta n_{1}$ and $\left.\Delta n_{2}\right)$, tilt measurements must be carried out in two instrumental faces (denoted with $I$ and $I I$ ).

Measurements in different instrumental faces become feasible through azimuthal rotation of the CCD-telescope by some rotation angle $\alpha$ (Fig 1a). Conventional DZCS measurements are carried out in opposite faces (i.e., $\alpha=180^{\circ}$ ), and the offsets can be conveniently removed through simple averaging of the tilt readings in the two faces. Because the rotation angle $\alpha$ will significantly deviate from $180^{\circ}$ for hexapod-based DZCS (say $\alpha=30^{\circ}$ to $60^{\circ}$ ), rigorous 
mathematical models (Hirt 2008) are used here that account for $\alpha$ in the reduction of the tilt measurements. Based on practical experiences, we assume that the tilt meters cannot be mounted exactly in perpendicular orientation. The shearing parameter $\varepsilon$ is therefore introduced to account for any misalignment of the two sensors from a $90^{\circ}$ angular difference between the two measurement axes (Fig. 1a). Typically, the shearing $\varepsilon$ is close to $90^{\circ}$, e.g., $89.4^{\circ}$, which is why power series expansions could be used where trigonometric functions of $\varepsilon$ appear in the sequel.
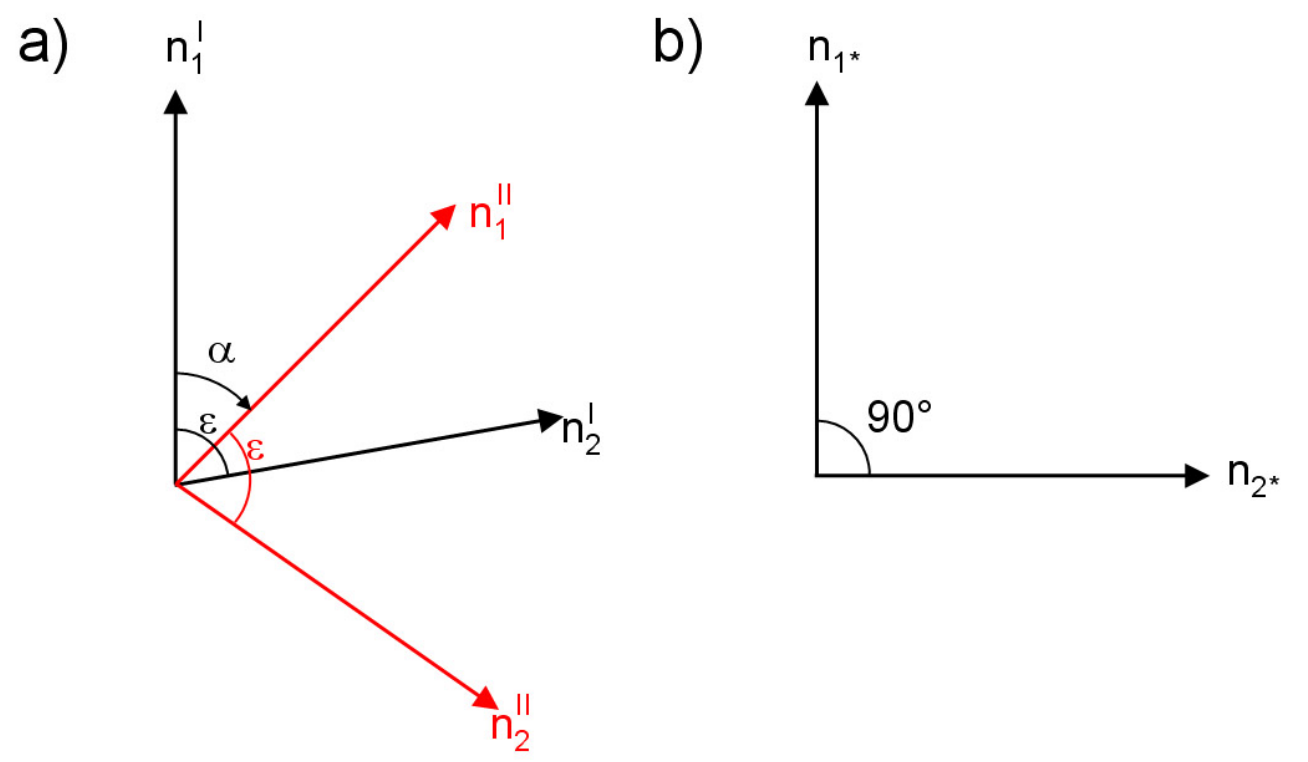

Figure 1. Panel a: Observation space for two-axes tilt measurements on a rotating platform. Black: measurement axes in face $I$, red: measurement axes in face II (situation after rotation by angle $\alpha$. In the observation space, the two axes are assumed to be sheared by angle $\varepsilon$. Panel b: Solution space with exactly perpendicular axes. Note that the direction of the first axis of the system $n_{1}$ in face $I$ is identical in both panels.

In the general case of tilt measurement on a DZCS, a set of two tilt readings $\left(n_{1}^{I}, n_{2}^{I}\right)$ is thus taken in face $I$, and another set in face II (tilt readings $n_{1}^{I I}, n_{2}^{I I}$ ), cf. Fig 1a. The four observations form the observation vector in the observation space 
$\mathbf{l}=\left(\begin{array}{c}n_{1}^{I} \\ n_{2}^{I} \\ n_{1}^{I I} \\ n_{2}^{I I}\end{array}\right)$

which is the input for the computation of the unknown CCD-telescope tilt $\left(n_{1^{*}}, n_{2^{*}}\right)-$ also denoted here as tilt correction - and the unknown sensor offsets ( $\left.\Delta n_{1}, \Delta n_{2}\right)$. The tilt corrections $\left(n_{1^{*}}, n_{2^{*}}\right)$ are free from the sensor offsets and refer to the orthogonal coordinate system shown in Fig. 1b. The two spatial directions in the solution space (Fig. 1b) are rigorously perpendicular (the shearing is removed), with the measurement axis of the first direction $\left(n_{1}\right)$ being identical in Figs. 1a and 1b. The vector of unknowns reads:

$\mathbf{x}=\left(\begin{array}{c}n_{1^{*}} \\ n_{2^{*}} \\ \Delta n_{1} \\ \Delta n_{2}\end{array}\right)$

The observations $\mathbf{I}$ and unknowns $\mathbf{x}$ are connected via the design matrix $\mathbf{A}$ which is set up as a function of the rotation angle $\alpha$ and the shearing $\varepsilon$ between the measurement axes (Hirt 2008):

$$
A=\left(\begin{array}{cccc}
1 & 0 & 1 & 0 \\
\cos \varepsilon & \sin \varepsilon & 0 & 1 \\
\cos \alpha & \sin \alpha & 1 & 0 \\
\cos (\alpha+\varepsilon) & \sin (\alpha+\varepsilon) & 0 & 1
\end{array}\right)
$$

The design matrix connects observations and unknowns linearly

$\mathbf{l}=\mathbf{A x}$

allowing calculation of the vector of unknowns $\mathbf{x}$ via left-multiplication with the inverse design matrix:

$\mathbf{x}=\mathbf{A}^{-1} \mathbf{l}$ 
It is clear that the design matrix A becomes singular and Eq. 5 cannot be solved for the unknowns if there is no rotation $\left(\alpha=0^{\circ}\right.$ ) between the two faces (compare the first and third row in Eq. 3). The design matrix becomes more stable as the rotation angle $\alpha$ increases, with optimum conditions assumed for the opposite-faces situation $\left(\alpha=180^{\circ}\right)$. If tilt was measured in more than two instrumental faces (e.g., each differing by $120^{\circ}$ ) in azimuth, the model would be over-determined, requiring e.g., least-squares or other adjustment techniques (e.g., Moritz 1980). Because DZCS measurements in general and tilt measurements in particular are usually conducted in the two faces, the over-determined case is not further dealt with in this paper.

In practical applications, numerical estimates for the rotation angle $\alpha$ are obtained as difference of the astronomical azimuths of the CCD star images in face I and II. In case a rotation unit (e.g., bearing) is deployed in the DZCS, an angle sensor might be alternatively installed and used to provide information on $\alpha$. For the hexapod-based DZCS (without bearing), the rotation angle $\alpha$ could be worked out as a function of the length variation of the six actuators between the two faces and the hexapod's constructive constants (e.g., leg separation), cf. Conti et al. (1998).

Numerical values for the shearing parameter $\varepsilon$ are determined with the so-called celestial calibration procedure whereby tilt measurements are compared against angles from star images. To increase the sensitivity for the determination of $\varepsilon$, the DZCS is deliberately tilted in all sky directions by about 100-120“ with respect to the plumb line (see Hirt 2004, Hirt et al., 2010 for details). In the future, this procedure might be augmented (or replaced) by a hexapod-based in-situ-calibration of the tilt sensors. Comparison of tilt values derived from measured leg lengths with measured tilt values in a least-squares sense should be capable of providing accurate estimates for the shearing $\varepsilon$, and also of the tilt sensor's scale factors.

\section{Monte-Carlo (MC) simulation}

\subsection{Idea and scheme}

The Monte-Carlo (MC) simulation (e.g., Metropolis and Ulam 1949, Rubinstein and Kroese 2007) is a statistical simulation method which can be used to study the behaviour of 
mathematical models or physical systems. The basic idea is the generation of sequences of random input values, which are propagated through some formalism (here the mathematical model for tilt measurements, Section 2). This yields output sequences of a certain statistical distribution, which is then interpreted and further analysed, e.g., in terms of standard deviations. As an advantage of the MC technique over classical numerical or analytical error propagation techniques, many different scenarios can be easily investigated with the MC simulation. The MC technique is chosen in the sequel as the most versatile statistical simulation tool to propagate uncertainties for all quantities involved through the tilt reduction model without simplifications. The robustness of results is ensured through choosing sufficiently large sample sizes (e.g., 1000 repetitions in each simulation). For validation purposes, analytical error propagation is applied (albeit with some simplifications) in Appendix A, providing a valuable mutual check on the outcomes of the MC simulation. 


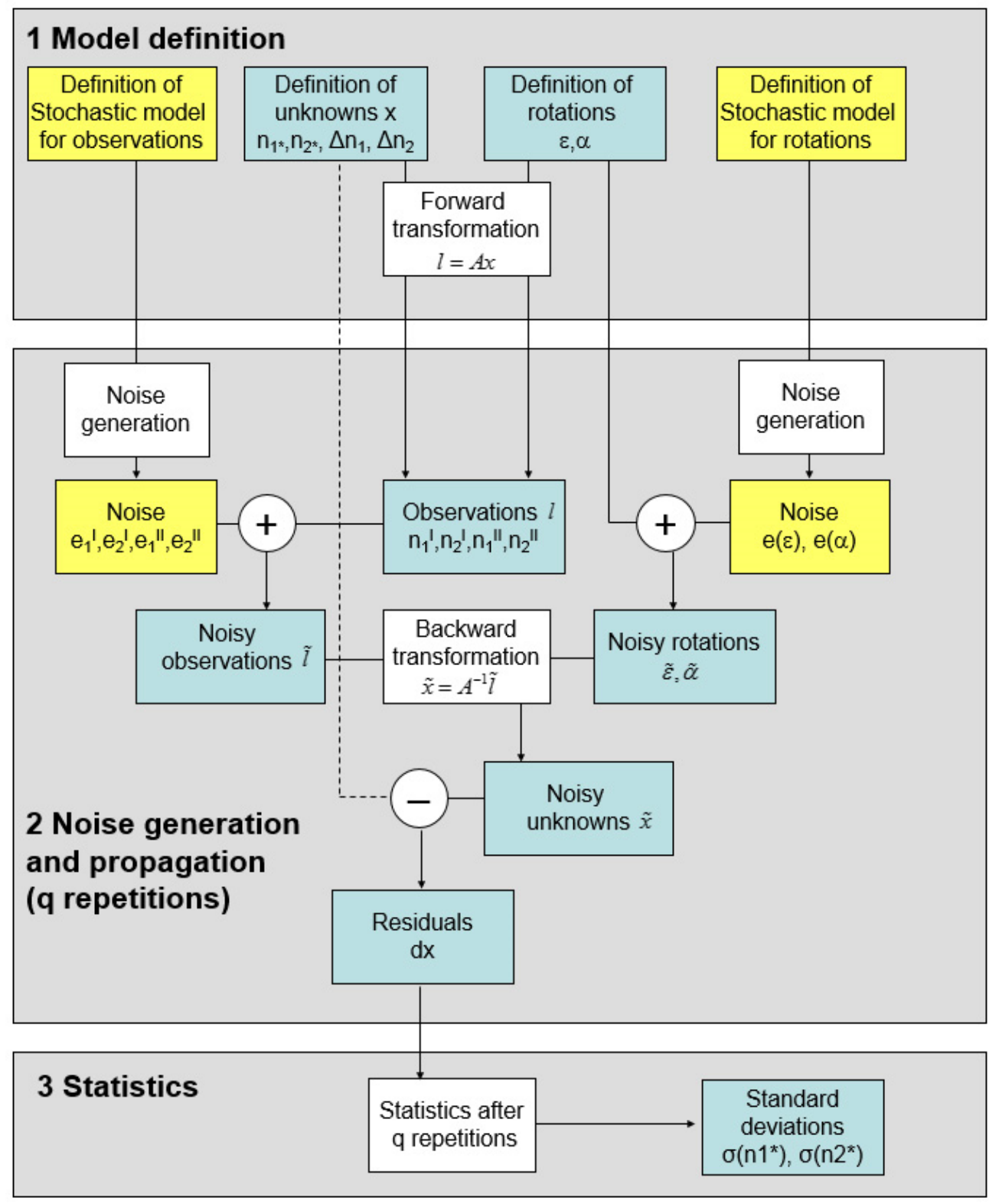

Figure 2. Flow diagram for the MC-simulation of tilt accuracy. White boxes indicate the operations applied.

We follow the MC simulation scheme shown in Fig. 2 to investigate the accuracy $\sigma\left(n_{1^{*}}\right)$, $\sigma\left(n_{2^{*}}\right)$ of the CCD-telescope tilt as a function of (1) the tilt $n_{1^{*}}, n_{2^{*}}$ itself, (2) the accuracy $\sigma\left(n_{1}^{I}\right), \sigma\left(n_{2}^{I}\right), \sigma\left(n_{1}^{I I}\right)$ and $\sigma\left(n_{2}^{I I}\right)$ of the tilt readings $n_{1}^{I}, n_{2}^{I}, n_{1}^{I I}$ and $n_{2}^{I I}$, (3) the shearing $\varepsilon$ and (4) related accuracy $\sigma(\varepsilon)$, and importantly, (5) the rotation angle $\alpha$ between the two faces and (6) its accuracy $\sigma(\alpha)$. The simulation results are insensitive for the sensor offsets $\left(\Delta n_{1}, \Delta n_{2}\right)$. 
The accuracy simulation starts by assigning numerical values to the CCD-telescope tilt ( $n_{1^{*}}$, $n_{2^{*}}$ ), the sensor offsets $\left(\Delta n_{1}, \Delta n_{2}\right)$, the shearing $\varepsilon$ and rotation angle $\alpha$, before applying the forward transformation (Eq. 4) to obtain the tilt meter readings $\left(n_{1}^{I}, n_{2}^{I}, n_{1}^{I I}, n_{2}^{I I}\right)$ the two sensors would measure if observation errors were absent. Regarding the numerical values, we make the following choices:

- The telescope tilt $\left(n_{1^{*}}, n_{2^{*}}\right)$ with respect to the plumb line is generally kept below 10“ (arc seconds) in DZCS field applications. However, the telescope tilt can be substantially larger, at the level of 100 “ when the DZCS is celestially calibrated. Both scenarios are simulated.

- The tilt values $\left(n_{1^{*}}, n_{2^{*}}\right)$ do not depend on the sensor offsets $\left(\Delta n_{1}, \Delta n_{2}\right)$. Given the simulated tilt accuracy is insensitive for $\left(\Delta n_{1}, \Delta n_{2}\right)$, we choose arbitrary numerical values of $\Delta n_{1}=-5$ “ and $\Delta n_{2}=+2$ “.

- The shearing $\varepsilon$ is generally close to $90^{\circ}$. Here we chose a value of $89.4^{\circ}$, which reflect results from DZCS calibrations (Hirt 2004).

- The rotation angle $\alpha$ is tested over a range from $1^{\circ}$ to $180^{\circ}$, which covers the expected range for hexapod-based rotations $\left(30^{\circ}\right.$ to $\left.60^{\circ}\right)$, while giving a complete picture of the role of $\alpha$ on the tilt accuracy.

In the next step, the stochastic models are defined for the observations $\mathbf{l}$ (Eq. 1) and for the rotations $\alpha, \varepsilon$. We assume Gaussian noise distribution for all six quantities.

- From practical experiences with high-resolution tilt meters, an observational accuracy of 0.04-0.05“ can be reached in field applications (Hirt and Kahlmann, 2004, Hirt et al., 2010). A value of 0.05 “ is assigned to the noise generator that produces sequences of random errors $e=\left(e_{1}^{I}, e_{2}^{I}, e_{1}^{I I}, e_{2}^{I I}\right)$ which follow the Gaussian distribution with the specified observational accuracy (Fig. 2). The vector of noisy observations $\tilde{\mathbf{l}}$ is then obtained through addition of random errors $e$ to the error-free tilt meter readings $\mathbf{I}$ (cf. Fig. 2). As noise generator, Matlab’s randn-function is used. 
- In a similar manner, Gaussian random noise is generated and added to the shearing $\varepsilon$ and rotation angle $\alpha$, yielding noisy values $\tilde{\varepsilon}$ and $\tilde{\alpha}$. The shearing $\varepsilon$ is assumed to be known with a standard deviation $\sigma(\varepsilon)=0.05^{\circ}$, and the rotation angle $\alpha$ with a standard deviation $\sigma(\alpha)=0.02^{\circ}$ (values from Hirt 2004).

We note that in the parameters $\alpha$ and $\varepsilon$ are determined via astronomical measurement procedures (rotation angle as difference between the astronomical azimuths of the CCD sensor in both faces, shearing via the celestial calibration). A further increase in accuracy may become possible through the development of a hexapod-based calibration procedure $(\varepsilon)$, and the determination of the rotation angle $\alpha$ from the actual hexapod leg lengths.

As central step of the MC simulation, the backward-transformation (Eq. 5) is applied to calculate noisy estimates for the telescope tilt $\left(\tilde{n}_{1^{*}}, \tilde{n}_{2^{*}}\right)$ from $\tilde{l}, \tilde{\varepsilon}$ and $\tilde{\alpha}$. This step "propagates" the generated noise from the observation space (vector l) into the solution space (vector $\mathbf{x})$. The noisy telescope tilt $\left(\tilde{n}_{1^{*}}, \tilde{n}_{2^{*}}\right)$ is then compared with the initial error-free values $\left(n_{1^{*}}, n_{2^{*}}\right)$, yielding residuals

$$
\begin{aligned}
& \delta n_{1^{*}}=\tilde{n}_{1^{*}}-n_{1^{*}} \\
& \delta n_{2^{*}}=\tilde{n}_{2^{*}}-n_{2^{*}}
\end{aligned}
$$

The described procedure is repeated $q$ times (here: $q=1000$, which was found to give statistically stable results from comparisons with other q-values, e.g., 100), giving sequences of residuals which are used to calculate standard deviations for the tilt corrections $\left(n_{1}^{*}, n_{2}^{*}\right)$

$$
\begin{aligned}
& \sigma\left(n_{1^{*}}\right)=\sqrt{\frac{\sum\left(\delta n_{1^{*}}\right)^{2}}{q}} \\
& \sigma\left(n_{2^{*}}\right)=\sqrt{\frac{\sum\left(\delta n_{2^{*}}\right)^{2}}{q}} .
\end{aligned}
$$

Exemplary histograms of the generated noise for the tilt observations $l$ and rotations $\alpha, \varepsilon$ are shown in Fig. 3a - c, and histograms for the derived telescope tilt $\left(\tilde{n}_{1^{*}}, \tilde{n}_{2^{*}}\right)$ in Fig. 3d. The 
data shown in Fig 3d is used to calculate the standard deviations $\sigma\left(n_{1^{*}}\right), \sigma\left(n_{2^{*}}\right)$ for further analysis.

a)

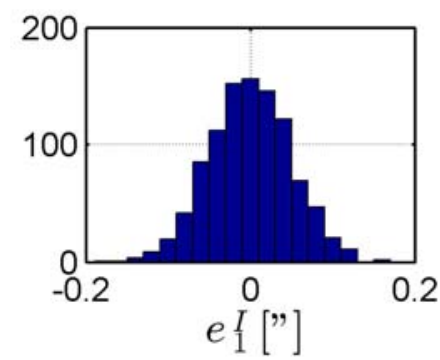

b)

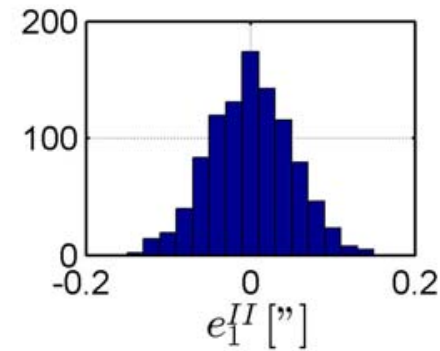

c)

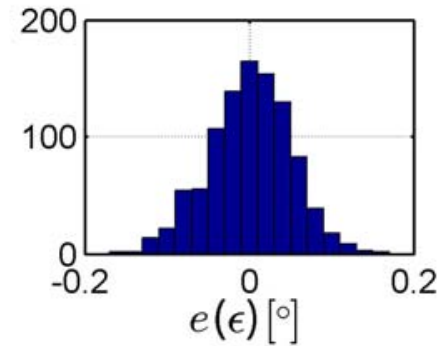

d)

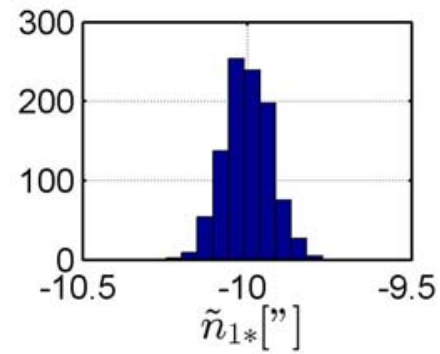

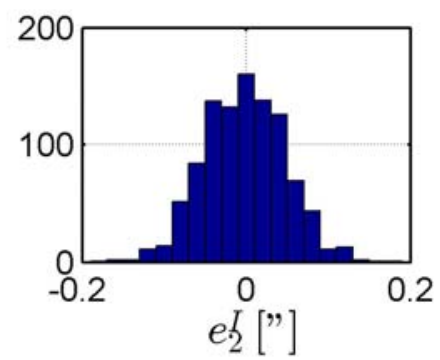
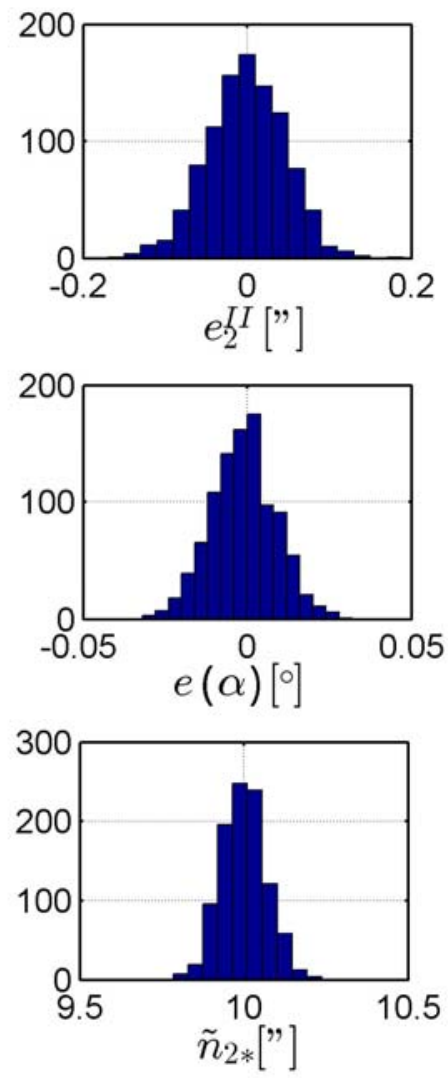

Figure 3. Histograms of sample data from the MC-simulation for $\alpha=60^{\circ}$. Panels a - b: histograms of the noise for the tilt measurements (in arc-seconds), panel c: histograms of the noise for the shearing $\varepsilon$ and rotation angle $\alpha$, panel d: histograms of noisy telescope tilt ( $\tilde{n}_{1^{*}}$, $\left.\tilde{n}_{2^{*}}\right)$.

\subsection{Results}

Figure 4 shows the Monte-Carlo simulated standard deviations $\sigma\left(n_{1^{*}}\right), \sigma\left(n_{2^{*}}\right)$ for the tilt corrections $\left(n_{1^{*}}, n_{2^{*}}\right)$ as a function of the rotation angle $\alpha$. The accuracies for both components of the tilt corrections are practically identical, and both deteriorate as the rotation 
angle $\alpha$ becomes smaller. The deterioration of the standard deviations $\sigma\left(n_{1^{*}}\right)$ as a function of the rotation angle $\alpha$ can be well modelled through a best-fitting (in a least-squares sense) analytical function

$$
\begin{aligned}
\sigma\left(n_{1^{*}}^{\text {fit }}\right)= & 0.0353^{“} \cdot \sin ^{-1}(0.5 \alpha) \approx \\
& \sigma\left(n_{1^{*}}\right) \frac{1}{\sqrt{2}} \cdot \sin ^{-1}(0.5 \alpha)
\end{aligned}
$$

which has been intuitively found based on the MC-results. The best-fitting analytical function is also shown in Fig. 4 (note that a very similar fit is obtained for $\sigma\left(n_{2^{*}}\right)$ ). With some simplifications of the tilt model (Eqs. 3-5), the Eq. (8) can also be derived analytically (see appendix A).

From Fig. 4, the accuracy $\sigma\left(n_{1^{*}}\right), \sigma\left(n_{2^{*}}\right)$ for the tilt corrections is about $0.036^{\text {“ }}$ when the tilt readings are taken in opposite faces with an observational accuracy of $\sigma\left(n_{1}^{I}\right)=\sigma\left(n_{2}^{I}\right)=$ $\sigma\left(n_{1}^{I I}\right)=\sigma\left(n_{2}^{I I}\right)=0.05$ “. This corresponds to a gain in accuracy by factor of about $\sqrt{2}$, which is plausible given that the two components $\left(n_{1^{*}}, n_{2^{*}}\right)$ of the tilt corrections are based on four observations $\mathrm{l}=\left(n_{1}^{I}, n_{2}^{I}, n_{1}^{I I}, n_{2}^{I I}\right)$. 


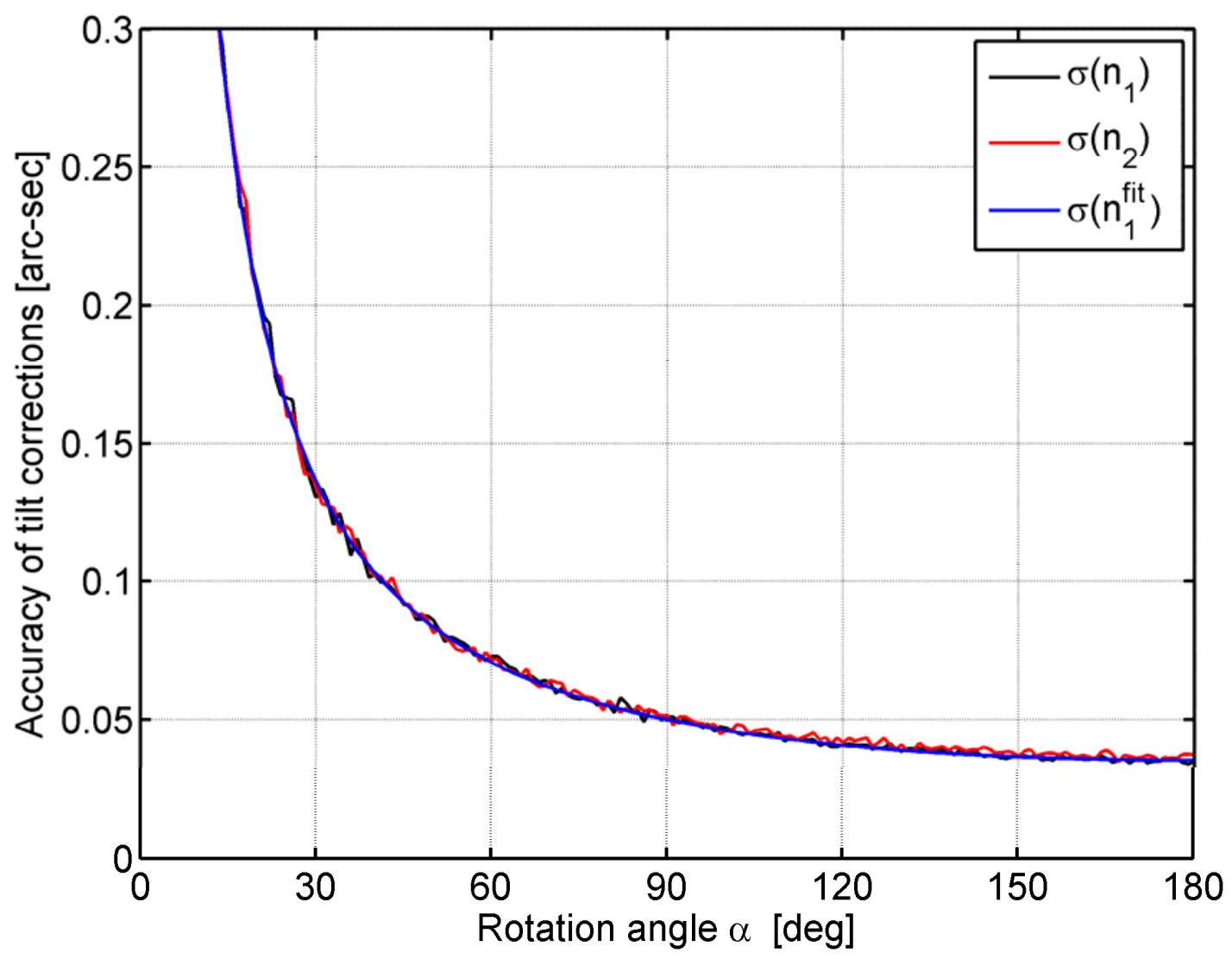

Figure 4. Simulated accuracy of tilt corrections $\left(n_{1^{*}}, n_{2^{*}}\right)$ as a function of the rotation angle $\alpha$ between instrumental faces. Simulation results is based on following parameters: $\left(n_{1^{*}}, n_{2^{*}}\right)$ $=\left(-10^{“}, 10^{“}\right), \varepsilon=89.4^{\circ}, \quad \sigma\left(n_{1}^{I}\right)=\sigma\left(n_{2}^{I}\right)=\sigma\left(n_{1}^{I I}\right)=\sigma\left(n_{2}^{I I}\right)=0.05^{\prime}, \sigma(\varepsilon)=0.05^{\circ}$ and $\sigma(\alpha)=0.02^{\circ}$.

For a reduced rotation angle $\alpha$ of $60^{\circ}$, the standard deviations $\sigma\left(n_{1^{*}}\right), \sigma\left(n_{2^{*}}\right)$ are at the level of 0.07 “, which corresponds to a deterioration by factor $\sim 2$ when compared to the conventional opposite-faces situation. A rotation angle of $30^{\circ}$ further reduces the accuracy by another factor of $\sim 2$ to $\sim 0.13-0.14$ “. A tilt correction accuracy at the 0.1 “-level requires a rotation angle of about $40-45^{\circ}$ or larger (Fig. 4).

Further insight into the expected accuracy of tilt corrections on a hexapod is obtained by varying the instrumental tilt $\left(n_{1^{*}}, n_{2^{*}}\right)$. While the tilt of the DZCS telescope is usually kept below 10“ during observation (this may require re-levelling if the DZCS is being operated at unstable sites), the tilt can be as large as 100-120“ during celestial calibration of the DZCS tilt sensors. Figure 5 shows the simulated standard deviations $\sigma\left(n_{1^{*}}\right), \sigma\left(n_{2^{*}}\right)$ as a function of the 
instrumental tilt $\left(n_{1^{*}}, n_{2^{*}}\right.$ ), and for the three cases $\alpha=30^{\circ}$ (top), $\alpha=60^{\circ}$ (centre), and $\alpha=$ $180^{\circ}$ (bottom).

The panels reveal that the standard deviations $\sigma\left(n_{1^{*}}\right), \sigma\left(n_{2^{*}}\right)$ may depend on the amplitudes of tilt corrections themselves to some certain extent. This behaviour reflects the propagation of the shearing parameter's and rotation angle's standard deviations $\sigma(\varepsilon)$ and $\sigma(\alpha)$ into the standard deviations $\sigma\left(n_{1^{*}}\right), \sigma\left(n_{2^{*}}\right)$ of the instrumental tilt. Fig. 5 shows that this effect diminishes for small tilt values (say few 10s of arc-seconds), while it becomes spurious when the instrumental tilt is large, at the level of 100 “. In the latter case, a deterioration in accuracy by a factor of $\sim 2$ becomes visible when the rotation angle is $30^{\circ}$ or $60^{\circ}$.
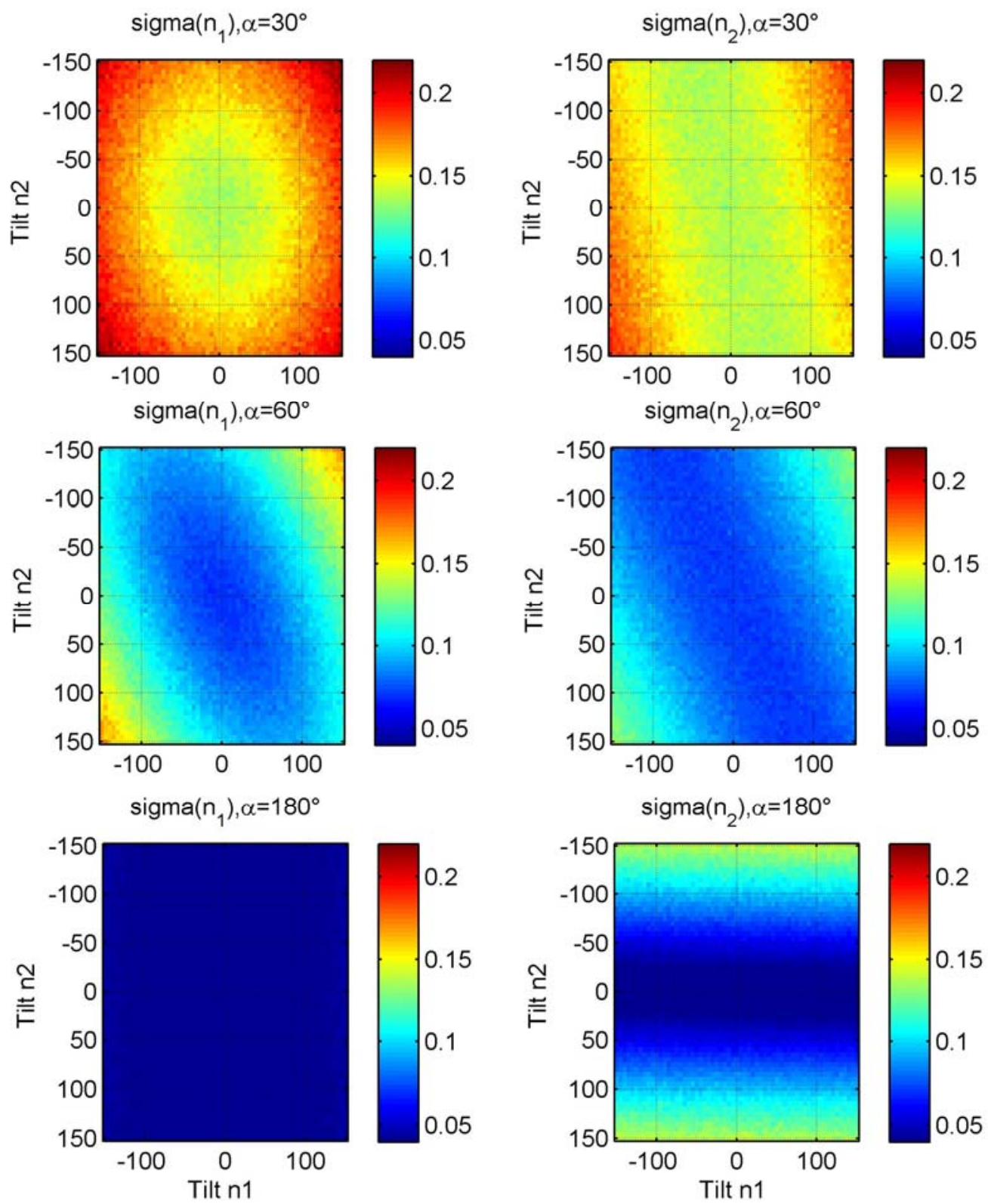
Figure 5. Simulated accuracy of tilt corrections $\left(n_{1^{*}}, n_{2^{*}}\right)$ as a function of the instrumental tilt $n_{1^{*}}, n_{2^{*}}$. Top: Rotation angle $=30^{\circ}$, Centre: rotation angle $=60^{\circ}$, Bottom: rotation angle $=$ $180^{\circ}$, left column: $\sigma\left(n_{1^{*}}\right)$, right column: $\sigma\left(n_{2^{*}}\right)$. Simulation results are based on following parameters: $\varepsilon=89.4^{\circ}, \quad \sigma\left(n_{1}^{I}\right)=\sigma\left(n_{2}^{I}\right)=\sigma\left(n_{1}^{I I}\right)=\sigma\left(n_{2}^{I I}\right)=0.05^{\prime}, \sigma(\varepsilon)=0.05^{\circ}$ and $\sigma(\alpha)$ $=0.02^{\circ}$. All units in arc-seconds.

By way of comparison, the conventional case $\left(\alpha=180^{\circ}\right)$ shows that the standard deviation $\sigma\left(n_{1^{*}}\right)$ is practically independent from the instrumental tilt $\left(n_{1^{*}}, n_{2^{*}}\right)$ over the whole range of tilt values shown. Different to this, $\sigma\left(n_{2^{*}}\right)$ is independent from $n_{1^{*}}$, but does depend on $n_{2^{*}}$, with a decrease in accuracy by factor 2-3 visible for $n_{2^{*}}$ values at the level of 100 “. This is related to the uncertainty of the shearing parameter $\sigma(\varepsilon)$ which propagates into $n_{2^{*}}$ (but not $n_{1^{*}}$ ) when observations are taken in opposite faces. Irrespective of the rotation angle investigated, the standard deviations are found to be invariant of the instrumental tilt if the DZCS telescope is well levelled (say better than 10“).

\section{Discussion and conclusions}

Other than conventional DZCS, the proposed hexapod-based DZCS is not capable of observing in opposite instrumental faces. Instead, the rotation angle of hexapod-based DZCS is limited to about $30-60^{\circ}$. A Monte-Carlo simulation study was performed to investigate the achievable accuracy for tilt corrections when the rotation angle is less than $180^{\circ}$. Statistical analysis of the tilt correction's accuracy as a function of the rotation angle revealed a loss in accuracy by factor of $\sim 2$ for $60^{\circ}$-rotation, and a factor of $\sim 4$ for $30^{\circ}$-rotation in comparison to the conventional $180^{\circ}$ rotation between opposite instrumental faces. This corresponds to a

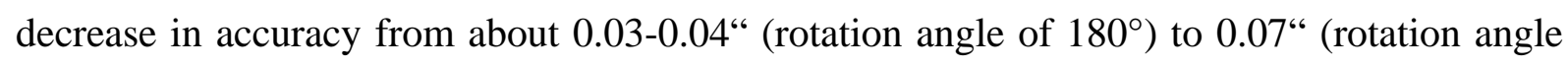
of $60^{\circ}$ ). For today's high-precision DZCS observations, it is reasonable to require a tilt correction accuracy at the 0.1 “-level or better. Our simulation shows that this requirement can be met with hexapod-based DZCS if the rotation angle is at least $40-45^{\circ}$, and $\sim 0.05^{\text {“-accurate }}$ tilt meters are deployed. 
Further to this, the MC-simulation study demonstrates that there is no loss in accuracy at all for rotation angles ranging between $\sim 150^{\circ}$ to $180^{\circ}$ compared to the opposite-faces situation. Thus, when using platforms with bearings or turntables, exact $180^{\circ}$-rotations between the instrumental faces are not required for optimum accuracies as long as the rotation angle is known with few $0.01^{\circ}$ accuracy and taken into account in the reduction of the tilt measurements (Section 2).

As a general conclusion of our study, a hexapod-based DZCS can be expected to be a suitable platform for accurate tilt measurements. While the accuracy for the instrumental tilt corrections will be worse in comparison to conventional opposite-face DZCS measurements, a satisfying accuracy level of 0.1 “ or better will be reachable on hexapod-based DZCS. As such, our study provides supporting evidence for feasibility of such a novel instrumentation.

These findings are not only relevant for the future development of hexapod-based DZCS, but also for the possible operation of other sensors (e.g., gravity meter) along with accurate tilt meters on hexapod platforms. Irrespective of the hexapod payload, a hexapod platform is expected to allow accurate determination of the platform tilt from measurement in two faces.

\section{Postscript}

A hexapod-based DZCS is now under development at the MTA (Magyar Tudományos Akadémia) Research Centre for Astronomy and Earth Sciences, based on a modified version of the Fly’s Eye hexapod described in Pál et al. (2013) and Vida et al. (2014).

\section{Acknowledgements}

Christian Hirt acknowledges funding from the Australian Research Council (DP120102441), and partial support by Technische Universität München (TUM) - Institute for Advanced Study (IAS), funded by the German Excellence Initiative and the European Union Seventh Framework Programme under grant agreement $n^{\circ}$ 291763. All authors thank the financial support of MTA CSFK which helped them to start their cooperation (Hungarian Academy of Sciences Grant LP2012-31). Willfried Schwarz is acknowledged for providing an alternative formulation of the mathematical tilt reduction model back in 2007. We thank two reviewers for their comments on the manuscript and the editor for handling of the review process. 


\section{Appendix}

\section{A Analytical derivation of the variance function of unknown parameters}

The expected value of the error of tilt corrections can also be estimated using the error propagation law (e.g. Moritz, 1980). The design matrix A (Eq. 3) connects the unknown parameters $\mathbf{x}$ (Eq. 2) with the actual tilt observations I (Eq. 1) through Eq. 4. Its solution based on the principles of least-squares adjustment involves the computation of the inverse of the so called normal matrix $\mathbf{N}$

$\mathbf{N}^{-1}=\mathbf{Q}=\left(\mathbf{A}^{T} \mathbf{P A}\right)^{-1}$,

where the weight matrix $\boldsymbol{P}=\boldsymbol{I}=\langle 1,1, \ldots, 1\rangle$, assumes that all the measurements have the same reliability. Eq. 9. is identical with so called co-factor matrix $\mathbf{Q}$ which is used to estimate the variance-covariance matrix $\mathbf{M}$ of the parameters $\mathbf{x}$

$\boldsymbol{M}=\sigma_{0}^{2} \boldsymbol{Q}$,

where $\sigma_{0}$ is the a posteriori RMS of a single measurement having unit weight in the non-overdetermined case. The elements (variances and covariances) of $\mathbf{Q}$ can be determined analytically if the design matrix is somewhat simplified to

$$
\boldsymbol{A}=\left(\begin{array}{cccc}
1 & 0 & 1 & 0 \\
0 & 1 & 0 & 1 \\
\cos \alpha & \sin \alpha & 1 & 0 \\
-\sin \alpha & \cos \alpha & 0 & 1
\end{array}\right)
$$

by using the approximations

$$
\begin{aligned}
& \cos \varepsilon \cong 0 \\
& \sin \varepsilon \cong 1 \\
& \cos (\alpha+\varepsilon)=-\sin \alpha \\
& \sin (\alpha+\varepsilon)=\cos \alpha
\end{aligned}
$$

This simplification assumes the axes of the tilt sensors are sufficiently perpendicular ( $\varepsilon \cong 90^{\circ}$ ). As further simplification in the analytical derivation, the angles $\alpha$ and $\varepsilon$ are assumed variance-free. In this case the normal matrix $\mathbf{N}$ becomes

$$
\mathbf{A}^{T} \mathbf{A}=\left(\begin{array}{cccc}
2 & 0 & 1+\cos \alpha & -\sin \alpha \\
0 & 2 & \sin \alpha & 1+\cos \alpha \\
1+\cos \alpha & \sin \alpha & 2 & 0 \\
-\sin \alpha & 1+\cos \alpha & 0 & 2
\end{array}\right)
$$


and for its inverse $\mathbf{Q}$ follows through analytical derivation

$$
\begin{aligned}
\mathbf{N}^{-1}=\mathbf{Q} & =\left(\begin{array}{cccc}
q^{2}\left(n_{1}^{*}\right) & q\left(n_{1}^{*}, n_{2}^{*}\right) & q\left(n_{1}^{*}, \Delta n_{1}\right) & q\left(n_{1}^{*}, \Delta n_{2}\right) \\
\cdot & q^{2}\left(n_{2}^{*}\right) & q\left(n_{2}^{*}, \Delta n_{1}\right) & q\left(n_{2}^{*}, \Delta n_{2}\right) \\
\cdot & \cdot & q^{2}\left(\Delta n_{1}\right) & q\left(\Delta n_{1}^{*}, \Delta n_{2}\right) \\
\cdot & \cdot & \cdot & q^{2}\left(\Delta n_{2}\right)
\end{array}\right) \\
& =\left(\begin{array}{cccc}
\frac{1}{1-\cos \alpha} & 0 & -\frac{1}{2} \operatorname{ctg}^{2} \frac{\alpha}{2} & \frac{1}{2} \operatorname{ctg} \frac{\alpha}{2} \\
0 & \frac{1}{1-\cos \alpha} & -\frac{1}{2} \operatorname{ctg} \frac{\alpha}{2} & -\frac{1}{2} \operatorname{ctg}^{2} \frac{\alpha}{2} \\
-\frac{1}{2} \operatorname{ctg}^{2} \frac{\alpha}{2} & -\frac{1}{2} \operatorname{ctg}^{\frac{\alpha}{2}} & \frac{1}{1-\cos \alpha} & 0 \\
\frac{1}{2} \operatorname{ctg} \frac{\alpha}{2} & -\frac{1}{2} \operatorname{ctg}^{2} \frac{\alpha}{2} & 0 & \frac{1}{1-\cos \alpha}
\end{array}\right),
\end{aligned}
$$

where $q$ denotes the weight coefficients. The elements of the variance-covariance matrix $\mathbf{M}$ finally provide the variances $\sigma^{2}$ and covariances $c$, i.e. of the unknowns

$\mathbf{M}=\sigma_{0}^{2} \mathbf{Q}=\left(\begin{array}{cccc}\sigma^{2}\left(n_{1}^{*}\right) & c\left(n_{1}^{*}, n_{2}^{*}\right) & c\left(n_{1}^{*}, \Delta n_{1}\right) & c\left(n_{1}^{*}, \Delta n_{2}\right) \\ . & \sigma^{2}\left(n_{2}^{*}\right) & c\left(n_{2}^{*}, \Delta n_{1}\right) & c\left(n_{2}^{*}, \Delta n_{2}\right) \\ . & . & \sigma^{2}\left(\Delta n_{1}\right) & c\left(\Delta n_{1}^{*}, \Delta n_{2}\right) \\ . & . & . & \sigma^{2}\left(\Delta n_{2}\right)\end{array}\right)$

With $\sigma_{0}=0.05^{\text {“ }}$ (observational accuracy), it follows for the standard deviation $\sigma\left(n_{1}^{*}\right)$ of the tilt correction:

$\sigma\left(n_{1}^{*}\right)=\sqrt{\sigma_{0}^{2} q^{2}\left(n_{1}^{*}\right)}=\sigma_{0}(1-\cos \alpha)^{-0.5}$

which confirms the results from the MC simulation (Eq. 8), recalling the theorem $1-\cos \alpha=2 \sin ^{2} 0.5 \alpha$. The very good agreement with the MC results implicitly shows that the uncertainties of the angles $\alpha$ and $\varepsilon$ play a negligible role for the standard deviation of the tilt correction in the present case. We finally note that the analytical derivation of (16) would become much more complicated if the above simplifications were not made. 


\section{References}

Abele M, Balodis, J, Janpaule I, Lasmane I, Rubans A and Zarinš, A 2012 Digital zenith camera for vertical deflection determination Geodesy and Cartography 38 123-129

Bürki B, Müller A and Kahle H-G 2004 DIADEM: The New Digital Astronomical Deflection Measuring System for Highprecision Measurements of Deflections of the Vertical at ETH Zurich Electronic Proc. IAG GGSM2004 Meeting, Porto, Portugal. Published also in: CHGeoid 2003, Report 03-33 A (ed. U. Marti et al.), Bundesamt für Landestopographie (swisstopo), Wabern, Switzerland

Chini R 2000 The Hexapod Telescope - A Never-ending Story. Reviews in Modern Astronomy 13, 257

Conti JP, Clinton CM, Zhang G and Wavering AJ 1998 Workspace variation of a hexapod machine tool, NISTIR 6135, National Institute of Standards and Technology, Available via:http://citeseerx.ist.psu.edu/viewdoc/download? doi=10.1.1.14.242

Csépány G, Pál A, Vida K et al 2014 The Fly's Eye Camera System -- an instrument design for large étendue time-domain survey Astronomical Society of the Pacific proceedings (submitted)

Halicioglu K, Deniz R and Ozener H 2012 Digital zenith camera system for Astro-Geodetic applications in Turkey Journal of Geodesy and Geoinformation 12 115-120 doi: 10.9733/jgg.131212.1

Hanada H, Araki H, Tazawa S et al. 2012 Development of a digital zenith telescope for advanced astrometry SCIENCE CHINA Physics, Mechanics \& Astronomy 55 4, 723-732, DOI: 10.1007/s11433-012-4673-1

Hirt C, Bürki B, Somieski A and Seeber G 2010 Modern Determination of vertical deflections using digital zenith cameras Journal Surveying Engineering 136 1, 1-12. DOI: 10.1061/ASCE_SU.1943-5428.0000009

Hirt C, Seeber G 2008 Accuracy Analysis of vertical deflection data observed with the Hannover Digital Zenith Camera System TZK2-D Journal of Geodesy 82 6, 347-356. DOI: 10.1007/s00190-007-0184-7

Hirt C and Flury J 2008 Astronomical-topographic levelling using high-precision astrogeodetic vertical deflections and digital terrain model data Journal of Geodesy 82 4-5, 231-248. DOI:10.1007/s00190-007-0173

Hirt C 2008 Zur Berücksichtigung von Scherung und Umschlagwinkel bei der Neigungsmessung mit zweiachsigen Neigungssensoren Zeitschrift für Vermessungswesen, 133 4, 266-273

Hirt C 2006 Monitoring and Analysis of Anomalous Refraction Using a Digital Zenith Camera System. Astronomy and Astrophysics 459 1, November III 2006: 283-290. DOI: 10.1051/00046361:20065485

Hirt C and Kahlmann T 2004 Hochpräzise Neigungsmessung mit dem elektronischen Pendelneigungssensor HRTM Zeitschrift für Vermessungswesen 129 4, 266-276 
Hirt C 2004 Entwicklung und Erprobung eines digitalen Zenitkamerasystems für die hochpräzise Lotabweichungsbestimmung. Wissenschaftliche Arbeiten der Fachrichtung Geodäsie und Geoinformatik an der Universität Hannover Nr. 253 (Dissertation), 188 pages.

Koch PM, Kesteven M, Nishioka H. et al. 2009 The AMIBA Hexapod Telescope Mount The Astrophysical Journal 694 2, 1670 doi:10.1088/0004-637X/694/2/1670

Kudrys J 2009 Automatic determination of the deflections of the vertical - first scientific results Acta Geodyn. Geomater. 6 3, 233-238

McCarthy DD 1976 Observations of the fortnightly nutation terms and the dynamical variation of latitude with photographic zenith tubes The Astronomical Journal 81 482-484

Moritz H 1980 Advanced physical geodesy. Abacus Press, Tunbridge Wells, Kent, p. 500.

Metropolis N and Ulam S 1949 The Monte Carlo Method. Journal of the American Statistical Association 44 247, 335-341. doi:10.2307/2280232

Ogrizovic V 2009 A construction of an advanced measuring system for astro-geodetic determinations, Publ. Astron. Obs. Belgrade 86145 - 150

Pál A, Mészáros L, Csépány G et al 2013 The Fly's Eye Camera System: An instrument design for large étendue time-domain survey Astron. Nachr. 334, 932-935. doi: 10.1002/asna.201211962

Rubinstein RY and Kroese DP 2007 Simulation and the Monte Carlo Method (2nd ed.). New York: Wiley \& Sons. ISBN 978-0-470-17793-8.

Runge C 1894 Über die Bestimmung der geographischen Breite auf photographischem Wege Zeitschrift für Vermessungswesen 23 300-304

Stewart, D 1965 A Platform with Six Degrees of Freedom Proceedings of the Institution of Mechanical Engineers 180 15, 371-386

Torge, W 2001 Geodesy (3 ${ }^{\text {rd }}$ edition), de Gruyter, Berlin, New York.

Vida K, Pál A, Mészáros L, Csépány G, Jaskó A, Mező G, Oláh K 2014 The Fly's Eye project: sidereal tracking on a hexapod mount. To appear in Proceedings Observing techniques, instrumentation and science for metre-class telescopes (workshop Tatranská Lomnica, Slovakia, September 23 - 26, 2013), available via eprint arXiv:1401.1719

Wang B, Tian L, Wang Z et al 2014 Image and data processing of digital zenith telescope (DZT-1) of China, Chin. Sci. Bull., online first, DOI 10.1007/s11434-014-0277-7 